\title{
Compressed Medicinal Gas Dosage Form
}

National Cancer Institute

\section{Source}

National Cancer Institute. Compressed Medicinal Gas Dosage Form. NCI Thesaurus.

Code C149656.

A gas packaged under pressure which is entirely gaseous at -50 degrees C. 